\title{
Rotenburger Allergie-Symposium
}

\begin{abstract}
An die 80 Ärzte der ÄDA-Regionalgruppe Niedersachsen-Nord nahmen am 25. August an der Fortbildung: „Neue Aspekte in der Allergie-Diagnostik" teil, die von T. Schaberg von der Lungenklinik Rotenburg-Unterstedt und R. Kroidl, Stade, im Diakonie-Krankenhaus Rotenburg an der Wümme perfekt organisiert worden war.
\end{abstract}

n seinem Referat „In-vitro-Tests in der Allergologie" stellte A. Bufe, Bochum, heraus, dass die Gesamt-IgE-Bestimmung für die angewandte Allergologie fast immer entbehrlich sei; nur zur Diagnostik und für das Monitoring der bronchopulmonalen Mykosen ließ er die Total-IgE-Bestimmung in der Routine gelten. Mehrere Zuhörer konnten sich dieser Ansicht nicht anschließen. IgG4Antikörper-Analysen wurden von Bufe für die tägliche Praxis ebenfalls sehr kritisch beurteilt sowie auch die meisten Histamin-Release-Tests.

J. Sennekamp, Bonn, ging in seinem Vortrag: „Häusliche Allergene - was gibt es außer der Hausstaubmilbe?" auf
Schafwolle, Wildseide, Birkenfeige, Latex und Tierhaare ein und betonte, dass Allergien auf diese ubiquitären Allergene oft nicht aus der Anamnese erfragt werden können. Daher sei es empfehlenswert, sie im Suchtest routinemäßig zu testen. Dass Allergien gegen Katzen - sofern zu Hause keine Katze gehalten wird - sich gut hyposensibilisieren lassen, war dem Publikum noch nicht allgemein bekannt.

Mit R. Kroidl, Stade, diskutierten wir nach seinem Vortrag: „Primäre und sekundäre Prävention in der Allergologie“, dass die Allergenkarenz im Prinzip immer hilfreich sei, dass aber nach neuesten Daten frühkindlicher Allergenkon-

\section{Allergica hat Premiere}

\section{Das Forum zum Thema Allergien und Atemwegserkran- kungen in Deutschland zu werden, mit diesem hohen Anspruch öffnet die Allergica von 24. bis 26. Mai 2002 erstmals ihre Tore.}

Die Voraussetzungen sind gut, denn die veranstaltende Messe Frankfurt hat es verstanden, alle mit dem Thema Beschäftigten Ärzte, Betroffene und Industrie - unter ein Messedach zu bringen.

Allergien sind die Epidemie des 21. Jahrhunderts, jeder dritte Deutsche leidet unter Asthma, Heuschnupfen oder Neurodermitis. Ein interdisziplinäres Forum zu schaffen, das sich sowohl an Ärzte als auch an Patienten richtet und das Thema „Allergien“ darüber hinaus stärker in die Öffentlichkeit trägt, scheint vor diesem Hintergrund nur logisch. "Ich erwarte, dass die Allergica“, so Chairman Prof. Dr. Karl Christian Bergmann, Bad Lippspringe, ,von allen Beteiligten als Chance begriffen wird, $\mathrm{zu}$ einer verbesserten medizinischen Versorgung der Betroffenen zu finden." Ihm zur Seite steht ein neunköpfiges Advisory Board, dem unter anderem
Prof. Dr. Dr. J. Ring, München, Prof. Dr. C. Bachert, Gent, und Prof. Dr. L. Klimek, Wiesbaden, angehören.

Der besondere Reiz der dreitägigen Allergica liegt in dem drei Ebenen übergreifenden Aufbau, bestehend aus dem medizinischen Fachkongress, der Fachausstellung und dem Informationsforum. Der Kongress mit seinen bisher 18 Symposien und die Ausstellung richten sich an Ärzte und medizinisches Fachpersonal. Das Informationsforum bietet den Betroffenen und ihren Familien die Möglichkeit, sich über ihre Erkrankung zu informieren und durch Patiententraining im Alltag besser mit ihrer Allergie zurecht zu kommen. takt, z. B. mit Katzen, vor Allergie schützen kann.

V. Kleine-Tebbe, Freiburg, stellte heraus, dass das „Vocal cord dysfunctionSyndrom“ Asthma vortäuscht. Die akuten Luftnotzustände, die selbst Intensivstationen „aufmischen “ können, werden oft fälschlicherweise mit Steroiden, $\beta$ Adrenergika und Theophyllin behandelt (vgl. S. 375 in diesem Heft).

Den Abschluss bildete der Dialog „Prick- versus Intracutan-Testung“ zwischen R. Kroidl und J. Sennekamp. Vielfach reicht der Prick-Test mit seiner geringen Sensitivität nicht aus. Insbesondere zur Diagnostik von isolierten Spätreaktionen ist deshalb der Intrakutan-Test grundsätzlich neben dem Prick-Test erforderlich. Im Publikum wurde die Erwartung an die Allergologen gestellt, dass der Patient nach initialem Prick-Test durch den Hausarzt vom Facharzt ggf. auch intrakutan getestet werde. Die noch nicht überall verbreitete intrakutane Testung mit 0,5-ml-Spritzen wurde favorisiert (Allergologie 1998; 21: 236-40). Prof. J. Sennekamp, Bonn

Ziel ist eine zielgruppenorientierte Ansprache von Arzt, Patient und Öffentlichkeit und die Integration aller relevanten Gruppen im Gesundheitswesen. Damit bietet sich die Chance, sinnvolle Kooperationen im Sinne der integrierten Versorgung zu schaffen - ganz im Sinne der Bundesgesundheitsministerin, die die Schirmherrschaft der Veranstaltung übernommen hat: „Allergien sind ein gesamtgesellschaftliches Problem, bei dem alle Beteiligten des Gesundheitswesens interdisziplinär zusammenwirken müssen, um nachhaltige Erfolge in Prävention und Behandlung zu erzielen."

\section{Hinweis}

Weitere Informationen sowie ein Symposiumsprogramm der Allergica sind erhältlich bei der:

Messe Frankfurt GmbH Ludwig-Erhard-Anlage 1 60327 Frankfurt am Main Tel. (o 69) 75 75-58 96 Fax (o 69) 75 75-65 33 www.allergica.de 